\title{
High-mass Star Formation towards the southern infrared Bubble S10
}

\author{
Swagat Ranjan Das ${ }^{1 *}$, Anandmayee Tej ${ }^{1}$, Sarita Vig ${ }^{1}$, Swarna Kanti Ghosh ${ }^{2}$ \\ C. H. Ishwara-Chandra ${ }^{2}$ \\ ${ }^{1}$ Indian Institute of Space Science and Technology, Trivandrum 695547, India \\ ${ }^{2}$ National Centre For Radio Astrophysics, Pune 411007, India
}

\begin{abstract}
We present a multi-wavelength analysis of the southern infrared bubble S10. Radio continuum emission is detected towards the bubble at 610 and $1280 \mathrm{MHz}$, and the bubble is estimated to be powered by massive stars of spectral type B0.5 - B0. A Class I/II type source, with an estimated mass of $6.2 \mathrm{M}_{\odot}$, lies $\sim 7^{\prime \prime}$ from the radio peak. Six massive clumps capable of forming massive stars are detected from the Herschel $250 \mu \mathrm{m}$ image. Modelling of the spectral energy distribution of these clumps indicates the presence of high luminosity, high accretion rate, massive young stellar objects possibly in the accelerating accretion phase. Further, the occurrence of a possible bow-wave towards the likely ionizing star is explored.
\end{abstract}

Keywords: stars: formation - ISM: H II region - ISM - radio continuum - ISM: individual objects (S10 - IRAS 17036-4033)

\section{Introduction}

High-mass stars $\left(\mathrm{M} \gtrsim 8 \mathrm{M}_{\odot}\right)$ are key objects which play a major role in the evolution of Galaxy. They are known to have a huge influence on the surrounding interstellar medium (ISM) through their radiative, mechanical, and chemical feedback. In the last decade, tremendous progress has been made towards the understanding of the interaction of massive stars with their surrounding ISM (see reviews by Zinnecker \& Yorke 2007; Tan et al. 2014). However, still, their properties are not completely understood, due to the challenges involved in both theory and observation of these objects.

Massive star formation is always accompanied by energetic UV photons. These energetic photons ionize the surrounding medium, creating H II regions (Wood \& Churchwell 1989; Churchwell 2002). $\mathrm{H}$ II regions grow or expand into their surrounding ISM, driven by several feedback mechanisms like thermal overpressure, powerful stellar winds, radiation pressure or the combination of all these sources (Churchwell et al. 2006; Deharveng et al. 2010; Simpson et al. 2012). The expansion of $\mathrm{H}$ II regions results in the formation of a spherical structure called "bubbles". These bubbles appear as a bright ring-like structure at mid- infrared (MIR) wavelengths, which traces the photodissociation (PDR) region of the bubbles (Weaver et al. 1977; Churchwell et al. 2006, 2007; Watson et al. 2008; Deharveng et al. 2010; Anderson et al. 2012; Kendrew et al. 2012; Ochsendorf et al. 2014 a,b). This

*email:dasswagat77@gmail.com

- "2nd Belgo-Indian Network for Astronomy \& astrophysics (BINA) workshop", held in Brussels (Belgium), 9-12 October 2018 


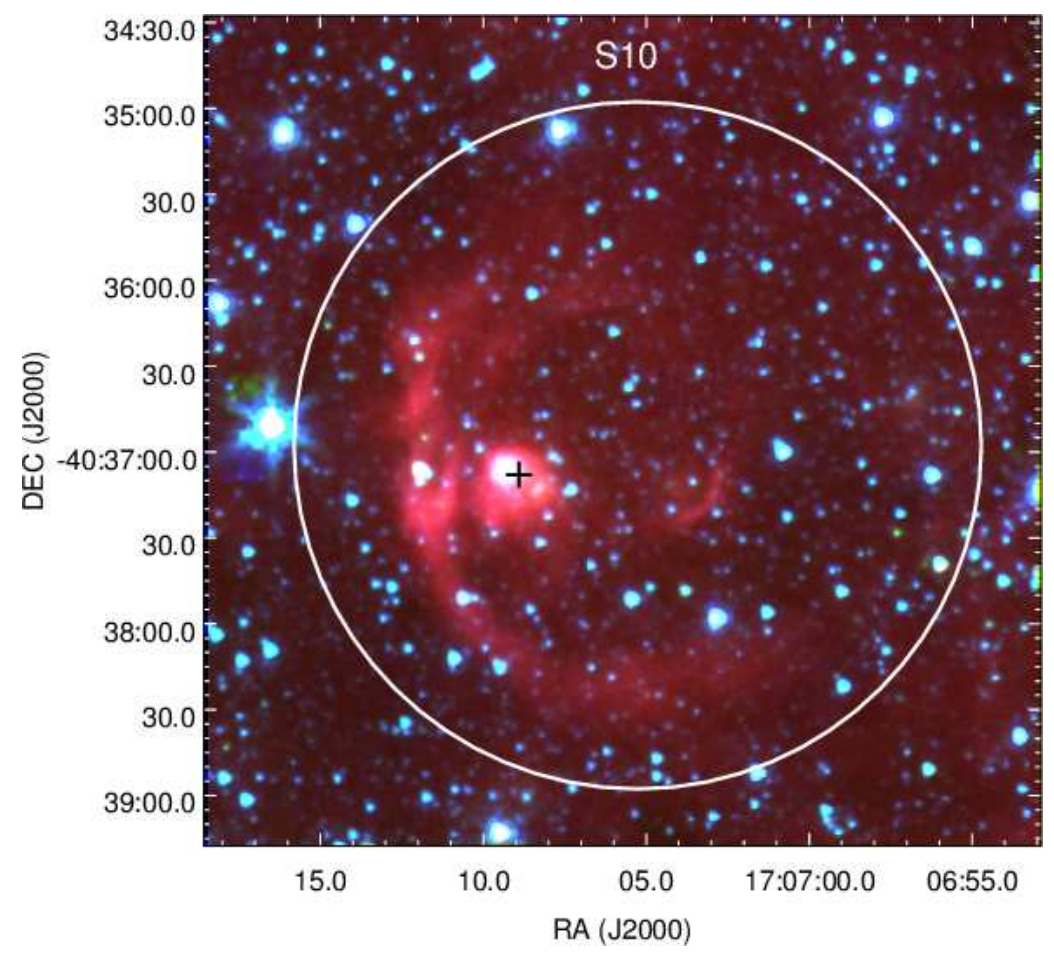

Figure 1: Colour composite image of bubble S10 with $8.0 \mu \mathrm{m}$ (red), $4.5 \mu \mathrm{m}$ (green) and $3.6 \mu \mathrm{m}$ (blue) colour coding. The ' + ' marks the positions of associated IRAS point source. Circle shows the studied regions.

bright emission of bubbles at MIR wavelengths is attributed to the polycyclic aromatic hydrocarbon (PAH) emission feature. In recent years these MIR bubbles have gained much attention to study the influence of massive stars on the surrounding ISM (Watson et al. 2008, 2009,2010). Based on the Spitzer-Galactic Legacy Infrared Mid-Plane Survey Extraordinaire (GLIMPSE ;Benjamin et al. 2003) $8 \mu \mathrm{m}$ emission, Churchwell et al. $(2006,2007)$ have catalogued $\sim 600$ infrared (IR) bubbles. Later on, a large number of such bubbles have been also identified in the Milky Way Project by Simpson et al. (2012).

In this work, we study a southern IR bubble S10, which is listed in Churchwell et al. (2006). Figure 1 shows the Spitzer-IRAC colour composite image of the region associated with S10. The MIR emission towards the bubble S10 displays a broken morphology, as mentioned in Churchwell et al. (2006). Such broken morphologies of bubbles are believed to be due to the non-uniform density of the ambient ISM and/or anisotropic stellar winds and radiation fields. S10 is also identified as a bubble in the Milky Way Project (Simpson et al. 2012). Morphologically S10 is elliptical as suggested by Churchwell et al. (2006) and nearly circular, as indicated by Simpson et al. (2012). However, the thickness $\left(0.98^{\prime}\right)$ estimated by Simpson et al. (2012) is on the higher side compared to the thickness $\left(0.3^{\prime}\right)$ determined by Churchwell et al. (2006). This bubble is associated with a bright IRAS source (IRAS 17036-4033), with a bolometric luminosity of $2.5 \times 10^{4} \mathrm{~L}_{\odot}$ (Beltran et al. 2006). Using the CS line velocity, Fontani et al. (2005) estimate the near and far kinematic distances for IRAS 17036-4033 to be 5.7 and $10.8 \mathrm{kpc}$, respectively. In this study, we aim to understand the effect of massive stars on the surrounding ISM and the star formation activity towards the IR dust bubble. With the multiwavelength data, we will be able to make a detailed analysis of the star formation activity associated with S10. 


\section{Observation and Data analysis}

To probe the ionized gas component associated with the bubble S10, we carried out radio continuum mapping at 610 and $1280 \mathrm{MHz}$ with the Giant Metrewave Radio Telescope (GMRT), Pune India on 17 and 20 July 2011. Details on the GMRT configuration can be found in Swarup et al. (1991). The log of the observation and radio maps are given in Table 1. Data reduction is performed with the Astronomical Image Processing System (AIPS) using standard procedures. More detail information regarding the different tasks of AIPS can be found in its cookbook available on the website of National Radio Astronomical Observatory (NRAO) ${ }^{1}$. Presence of bad data and RFI (radio frequency interference) are identified by careful visual inspection. Subsequently, all these bad data have been flagged. After proper flagging and calibration, clean image has been generated. The wide-field imaging technique has been employed to account for the $w$-term effects. This $w$-term effect is important to account for the curvature of the sky. Several iterations of self calibrations have been made to reduce the amplitude and phase errors and obtain better rms noise in the maps. Final maps are primary beam corrected and a temperature correction has been applied to account for the contribution of Galactic diffuse emission.

Table 1: Details of the radio interferometric continuum observations.

\begin{tabular}{lll}
\hline \hline Details & $610 \mathrm{MHz}$ & $1280 \mathrm{MHz}$ \\
\hline Date of Obs. & 17 July 2011 & 20 July 2011 \\
Flux Calibrators & 3C286,3C48 & 3C286,3C48 \\
Phase Calibrators & $1626-298$ & $1626-298$ \\
Synth. beam & $14.4^{\prime \prime} \times 8.5^{\prime \prime}$ & $8.8^{\prime \prime} \times 4.4^{\prime \prime}$ \\
Position angle. (deg) & 10.61 & 15.02 \\
rms noise (mJy/beam) & 0.7 & 0.2 \\
Int. Flux (mJy) & 203 & 44 \\
(integrated upto 3 $\sigma$ level) & & \\
\hline
\end{tabular}

In this work, apart from the radio continuum observations, various archival data are also used. We have used MIR and Far-infrared (FIR) data obtained from the archives of Spitzer ${ }^{2}$ and Herschel $^{3}$ Space Telescopes, respectively. MIR data are used to study the population of young stellar objects (YSOs) and warm dust associated with the region. The FIR data are used to study the physical properties of cold dust emission associated with the region.

\section{Results and Discussion}

\subsection{Nature of ionized emission and YSOs}

Figure 2 shows the radio continuum maps as contours plotted on the $8 \mu \mathrm{m}$ image. The region associated with S10 shows the presence of faint diffuse ionized emission mostly distributed in the second quadrant of the bubble. The $610 \mathrm{MHz}$ emission displays a relatively steep density gradient with enhanced emission towards the likely centre of the bubble and a more extended emission towards the north-east. However, the higher frequency map at $1280 \mathrm{MHz}$ is seen to be less extended in the southeast and north-west direction but follows the general morphology seen at $610 \mathrm{MHz}$. Assuming the

\footnotetext{
${ }^{1} \mathrm{http}: / /$ www.aips.nrao.edu/CookHTML/CookBook.html

${ }^{2}$ https://irsa.ipac.caltech.edu/Missions/spitzer.html

${ }^{3}$ https://irsa.ipac.caltech.edu/Missions/herschel.html
} 

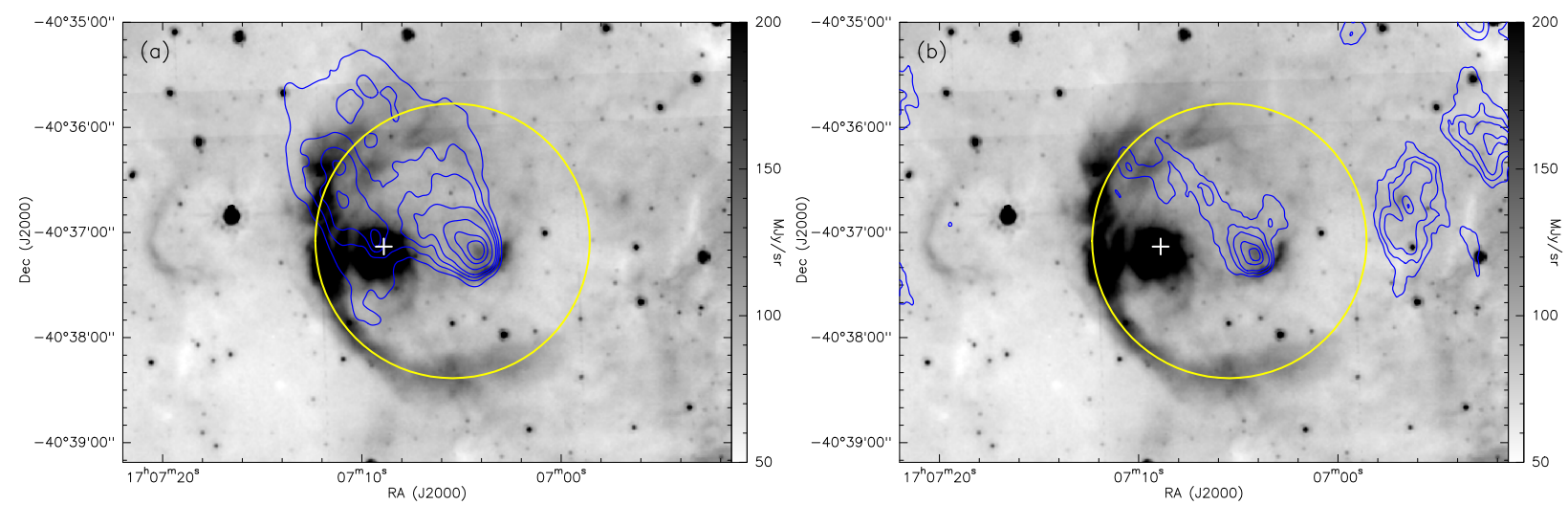

Figure 2: Radio continuum emission probed in S10 are over plotted on the IRAC $8.0 \mu \mathrm{m}$ images. (a) $610 \mathrm{MHz}$ map of the region associated with S10. The contour levels are 3, 3.5, 4.0, 4.5, 5.5, 6.5, 7 times $\sigma(0.7 \mathrm{mJy} / \mathrm{beam})$. (b) $1280 \mathrm{MHz}$ of the region associated with $\mathrm{S} 10$. The contour levels are 3, 4, 5, 7, 8 times $\sigma(0.2 \mathrm{mJy} /$ beam $)$. The circle and '+' mark are same as in Figure 1.

ionized emission at $1280 \mathrm{MHz}$ to be free-free and optically thin in nature, we estimate the zero-age main-sequence (ZAMS) spectral type of the ionizing source(s) to lie between B0.5 - B0. This estimate should be considered as the lower limit since we assume the emission to be optically thin. However, the emission could be optically thick at $1280 \mathrm{MHz}$. Also, we have not taken into account the absorption of Lyman continuum photons by dust.

To understand the nature of the stellar population and star-formation activity towards the bubble S10, we have identified young stellar objects (YSOs). To identify and classify YSOs, we have used the photometric data obtained from the archive of Spitzer-GLIMPSE survey. Using various color criteria (Allen et al. 2004; Gutermuth et al. 2008; Simon et al. 2007) and using the MIR photometry, we have identified 14 YSOs in the region associated with S10. Towards the radio peak, there is a red NIR source (hereafter IRS1) $\left(\alpha_{2000}=17: 07: 03.60, \delta_{2000}=-40: 37: 10.70\right)$ with colours $\mathbf{J}-\mathrm{H}=$ 2.44 and $\mathrm{H}-\mathrm{K}=1.67$. From the YSO classification scheme, the source IRS1 is classified as Class II YSO. Further, to quantify the nature of IRS1, we have carried out Spectral Energy Distribution (SED) modelling using the online SED fitting tool of Robitaille et al. (2007). For SED fitting we have used fluxes from 2MASS, Sptizer and Herschel bands along with the 870 and $1200 \mu \mathrm{m}$ bands. IRS1 has been identified as a point source in 2MASS, and three IRAC bands (3.6, 4.5, and $5.8 \mu \mathrm{m})$ but not in $8 \mu \mathrm{m}$ band. However, visually, IRS1 appears as a point source in $8 \mu \mathrm{m}$ image, and we have estimated the flux by carrying out photometry using IRAF. Using a clump aperture of effective diameter $\sim 12^{\prime \prime}$, we have estimated the flux from FIR images. Assuming that IRS 1 is associated with the bubble S10, we have used a distance range of 5.5 to $5.9 \mathrm{kpc}$ in the model fitting tool. As discussed earlier, IRS1 is a reddened source, and its location in the JHK CCP (not presented in the paper) gives an estimate of $A_{v} \sim 15$ mags. Hence, for the model fitting we use a range of $A_{v}$ from 1 to 20 mags. This SED modelling suggested that the source IRS1 be an intermediate star with an estimated mass of $6.2 \mathrm{M}_{\odot}$ (estimated from the best fit model). Therefore IRS1 is unlikely to be the NIR counterpart of the exciting star responsible for the ionized emission. It is possible that the massive ionizing star is deeply embedded and does not reveal itself in the NIR.

\subsection{Nature of cold dust emission}

Pixel-wise, modified blackbody fits to the thermal dust emission using Herschel FIR data is performed to construct dust temperature and column density maps (Das et al. 2017, 2018). To generate the 
maps, we have used the Herschel 160, 250, 350, and $500 \mu \mathrm{m}$ band images. We have excluded the $70 \mu \mathrm{m}$ band map since this band has contribution from both warm and cold dust. The generated dust temperature and column density maps are shown in Figure 3. Dust temperature is higher ( $23 \mathrm{~K}$ ) towards the IRAS point source. Overlying the radio continuum emission on temperature map, it is clear that the ionized regions are traced by warmer dust component compared to the other regions. The column density map for the region associated with S10 shows a high-density elongated clump towards the south-west of the bubble mostly outside the periphery. A high-density region is also seen stretching in the south-east and north-west direction on the opposite boundary.
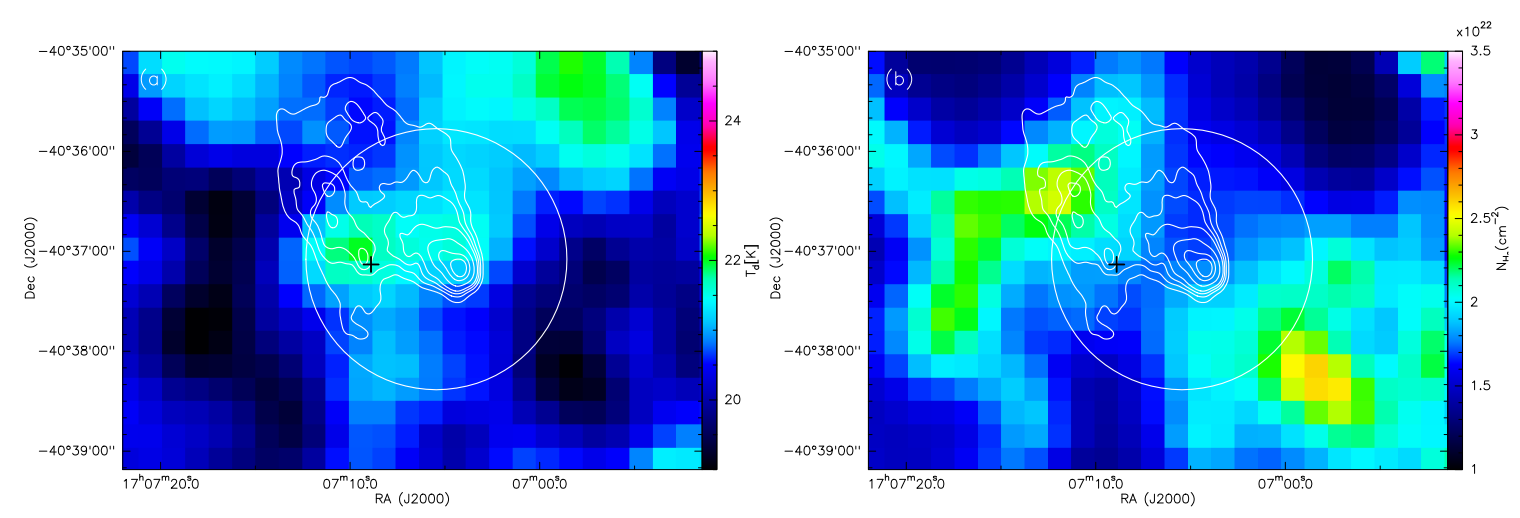

Figure 3: (a) Dust temperature map and (b) column density map of region associated with S10. The radio emission at $610 \mathrm{MHz}$ is also shown as contours with the same levels as in Figure 2. The circle and ' + ' symbols are same as in Figure 1.

Six cold dust clumps are identified in the region associated with the bubble S10, from Herschel $250 \mu \mathrm{m}$ image, using the 2D clumpfind algorithm (Williams et al. 1994). Following procedures explained by Kauffmann et al. (2008) and Das et al. (2017), we have estimated various physical properties of the dust clumps. Mass and linear diameter of the dust clumps vary in the range of 350 $-1400 \mathrm{M}_{\odot}$ and $0.7-1.1 \mathrm{pc}$, respectively. In order to understand the nature of the sources towards these clumps, we use the online SED model fitting tool of Robitaille et al. (2007) to fit the clump fluxes with the inbuilt YSO models. This is along the lines discussed in Zavagno et al. (2010). Here, we assume that each clump would produce a single high-mass star. For model fitting, we have used clump fluxes from 24, 70, 160, 250, 350, 500, 870, and $1200 \mu \mathrm{m}$ bands. From the SED modelling these clumps show that they harbor high luminosity, high envelope accretion rate, massive YSOs. The empirical mass-radius relation suggested by Kauffmann \& Pillai (2010), defined a threshold for clouds to form massive stars. Using this empirical mass-radius relation, we found that all the dust clumps are potential to form high-mass stars. Following the discussions made in Molinari et al. (2008), we have tried to understand the evolutionary nature of the dust clumps. The relation of the luminosity of the star and the envelope mass show that all the clumps are in accelerating accretion phase. Further, we have plotted the clump mass versus the efficiency of the clump (shown in Figure 4), to form the most massive star. From this plot, we found that the efficiency decreases, with the increase in clump mass. This can be understood with the fact that massive stars always form in a cluster along with both low and intermediate mass stars. The larger the clump mass, the more populous would be the cluster and hence, relatively less mass goes to the most massive member. Hence, using the mass of the most massive member understates the star-forming efficiencies. 


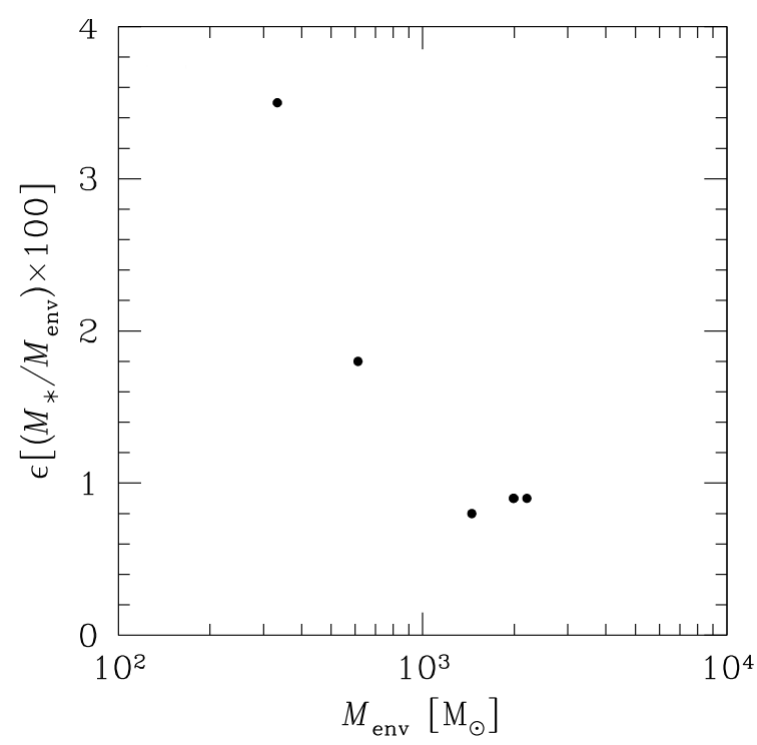

Figure 4: Plot of the star forming efficiency of the clumps as a function of the envelope mass. Efficiency of stars are calculated as $\epsilon=\left[\frac{M_{*}}{M_{\text {env }}} \times 100\right]$, where $M_{*}$ is the final mass of star and $M_{\text {env }}$ is the envelope mass of clump as obtained from the SED fitting.

\section{Possible Bow-wave in S10?}

It is believed that several feedback mechanisms play significant roles in the formation of the bubbles. The traditional picture of the formation of bubbles with the help of stellar wind is explained in Weaver et al. (1977). However, this picture of bubble formation lacks observational support, as outlined in Ochsendorf et al. (2014a). The wind-blown model of the bubble failed to explain the observational challenges such as the non-detection of X-ray emission inside the bubble and the presence of dust in the H II regions (Ochsendorf et al. 2014a). The view that evaporation of dense cloudlets replenishes the interior of bubbles with the new generation of dust grains could explain the presence of dust seen in the H II regions associated with the bubbles (Everett \& Churchwell 2010). This mechanism, however, fails to account for the growing evidence of arc-type structures seen at $24 \mu \mathrm{m}$ in the interior of bubbles and the observation of incomplete shells in H II bubbles (Deharveng et al. 2010; Kang et al. 2009; Watson et al. 2008).

In Figure 5, we show the three colour composite image of S10 using $8 \mu \mathrm{m}$ (Spitzer - IRAC), $24 \mu \mathrm{m}$ (Spitzer - MIPS) and $610 \mathrm{MHz}$ (GMRT). The $8 \mu \mathrm{m}$ emission is seen as a prominent outer shell and an inner arc-type feature as mentioned earlier. The $24 \mu \mathrm{m}$ emission shows enhanced distribution mostly in three localized regions. This interesting morphology prompted us to investigate the presence of a bow-wave but at shorter wavelengths compared to the $24 \mu \mathrm{m}$ arcs discussed in Ochsendorf et al. $(2014 a, b)$. The likely ionizing star responsible for S10 falls in the 'weak-wind' category with an estimated $\log \left(\mathrm{L} / \mathrm{L}_{\odot}\right)$ lying between 4.04 (B0.5) and 4.40 (B0). Using the formulations discussed in Mac Low et al. (1991), we have calculated the stand-off distance (explained as distance of dust from star, where the momentum flux of the stellar wind equates with the ram pressure of the star moving through the ISM) $r_{s}$ to be $0.3^{\prime \prime}-0.5^{\prime \prime}$ for spectral type of B0.5 - B0 estimated for the ionizing star of S10. From the 5.8 and $8 \mu \mathrm{m}$ images we estimate the arc to be at a distance $\left(r_{\text {min }}\right)$ of $\sim 15^{\prime \prime}$ from the radio peak which corresponds to $\sim 0.4 \mathrm{pc}$, far exceeding the stand-off distance, $r_{s}$. Here, we assume the position of radio peak to be the location of the ionizing star. This is consistent with what is expected for a bow-wave to occur. In Ochsendorf et al. (2014a), a similar dust structure qualifying 
as a dust-wave is seen at a distance of $0.1 \mathrm{pc}$ from $\sigma$ Orionis AB. However, a more detailed analysis of the dust grain characteristics and its wavelength dependence is necessary before we can address the possibility of occurrence of a bow-wave conclusively at shorter MIR wavelengths.

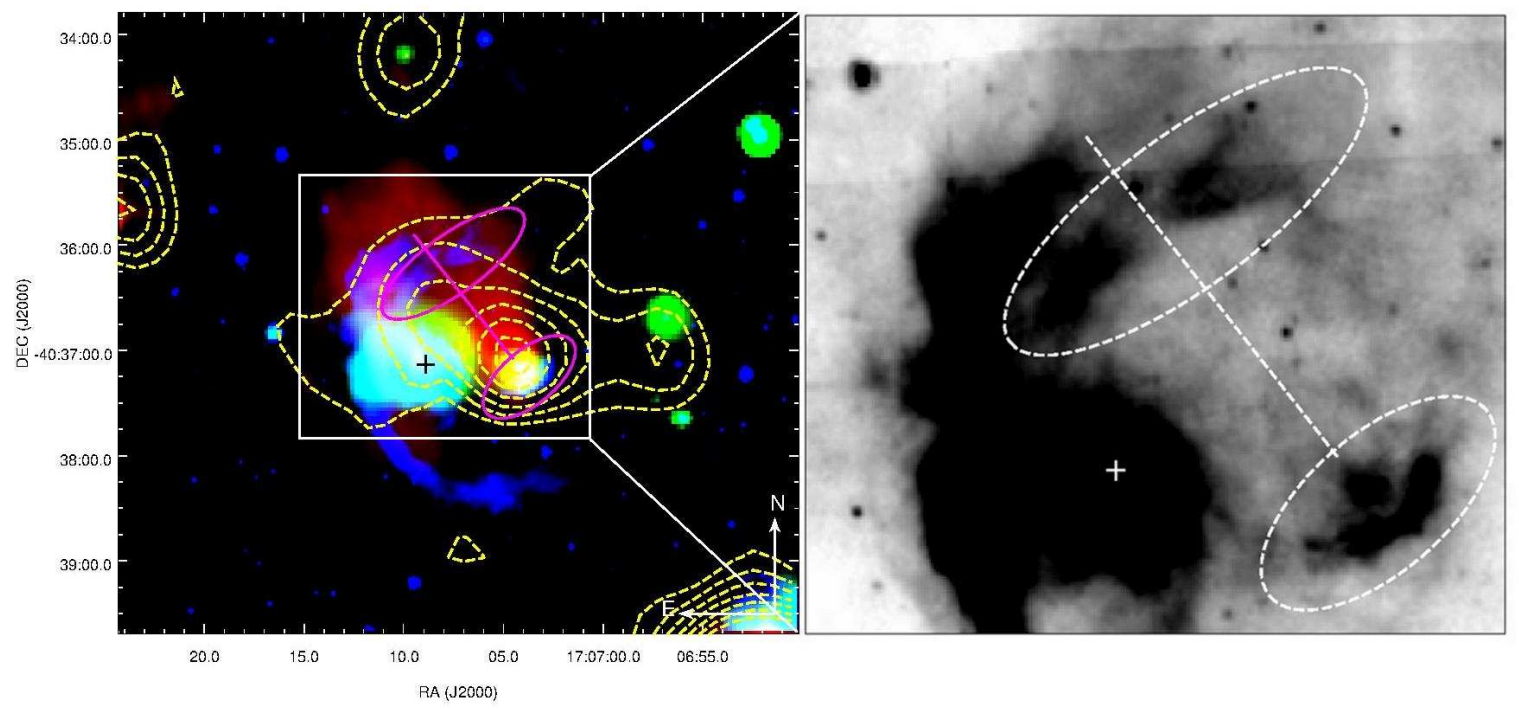

Figure 5: Left panel: Three-color composite image of the region associated with the bubble S10 with $8 \mu \mathrm{m}$ Spitzer-GLIMPSE (blue), $24 \mu \mathrm{m}$ MIPSGAL (green), and $610 \mathrm{MHz}$ GMRT (red). Low resolution radio emission at $843 \mathrm{MHz}$ from SUMSS is shown as contours. Right panel: Enlarged view of the outer shell rupture and the arc-type feature in the $8 \mu \mathrm{m}$ image. This figure is adopted from Ranjan Das et al. (2016).

\section{Summary}

In this work we have done a multi-wavelength study towards southern IR bubble S10. The radio maps show emission in the interior of the bubble. The radio peak is seen to be located at the likely center of the bubble. The spectral type of the ionizing source responsible for this emission is estimated to be B0.5 - B0. The source IRS1, which lies close to the radio peak is unlikely to be the NIR counterpart of the ionizing source. Six massive dust clumps are found to be associated with the bubble. From the mass-radius relation, these dust clumps show potential nature to form high-mass stars. The possible formation of a bow-wave at shorter wavelength has also been investigated.

\section{Acknowledgements}

We thank the staff of the GMRT, that made the radio observations possible. GMRT is run by the National Centre for Radio Astrophysics of the Tata Institute of Fundamental Research. We thank R. Cesaroni for providing the $1.2 \mathrm{~mm}$ maps of the regions. This work made use of data products from the Sydney University Molonglo Sky Survey (SUMSS) archives and Apex Telescope under the Apex Telescope Large Area Survey of the Galaxy (ATLASGAL). The ATLASGAL project is a collaboration between the Max-Planck-Gesellschaft, the European Southern Observatory (ESO) and the Universidad de Chile. 


\section{References}

Allen L. E., Calvet N., D’Alessio P. et al. 2004, ApJS, 154, 363

Anderson L. D., Zavagno A., Deharveng L. et al. 2012, A\&A, 542, A10

Beltran M. T., Brand J., Cesaroni R. et al. 2006, A\&A, 447, 221

Benjamin, R. A., Churchwell E., Babler B. L. et al. 2003, PASP, 115, 953

Churchwell E. 2002, ARA\&A, 40, 27

Churchwell E., Povich M. S., Allen D. et al. 2006, ApJ, 649, 759

Churchwell E., Watson D. F., Povich M. S., 2007, ApJ, 670, 428

Deharveng L., Schuller F., Anderson L. D. et al. 2010, A\&A, 523, A6

Das S. R., Tej A., Vig S. et al. 2017, MNRAS, 472, 4750

Das S. R., Tej A., Vig S. et al. 2017, A\&A, 612, A36

Everett J., Churchwell E. 2010, in ASP Conf. Ser. 438

Fontani F., Beltran, M. T., Brand J. et al. 2005, A\&A, 432, 921

Gutermuth R. A., Myers P. C., Megeath S. T. et al. 2008, ApJ, 674, 336

Kang M., Bieging J. H., Kulesa C. A., Lee Y. 2009, ApJ, 701, 454

Kauffmann J., Bertoldi F., Bourke T. L., Evans N. J. II, Lee C. W. 2008, A\&A, 487, 993

Kauffmann J., Pillai T. 2010, ApJ, 723, L7

Kendrew S., Simpson R., Bressert E. et al. 2012, ApJ, 755, 71

Mac Low M.-M., van Buren D., Wood D. O. S., Churchwell E. 1991, ApJ, 369, 395

Molinari S., Pezzuto S., Cesaroni R. et al. 2008, A\&A, 481, 345

Ochsendorf B. B., Cox N. L. J., Krijt S. et al. 2014a, A\&A, 563, A65

Ochsendorf B. B., Verdolini S., Cox N. L. J. et al. 2014b, A\&A, 566, A75

Ranjan Das S., Tej A., Vig S., Ghosh S. K., Ishwara Chandra C. H. 2016, AJ, 152, 152

Robitaille T. P., Whitney B. A., Indebetouw R., Wood K. 2007, ApJS, 169, 328

Simon J. D., Bolatto A. D., Whitney B. A. et al. 2007, ApJ, 669, 327

Simpson R. J., Povich M. S., Kendrew S. et al. 2012, MNRAS, 424, 2442

Swarup G., Ananthakrishnan S., Kapahi V. K. et al. 1991, CSci, 60, 95

Tan J. C., Beltran M. T., Caselli P. et al. 2014, in Beuther H., Klessen R. S., Dullemond C. P., Henning T., eds, Protostars and Planets VI, University of Arizona Press, Tucson, 149

Watson C., Povich M. S., Churchwell E. B. et al. 2008, ApJ, 681, 1341

Watson C., Corn T., Churchwell E. B. et al. 2009, ApJ, 694, 546

Watson C., Hanspal U., Mengistu A. 2010, ApJ, 716, 1478

Weaver R., McCray R., Castor J., Shapiro, P., Moore R. 1977, ApJ, 218, 377

Williams, J. P., de Geus, E. J., Blitz, L. 1994, ApJ, 428, 693

Wood D. O. S., Churchwell E. 1989, ApJS, 69, 831

Zavagno A., Anderson L. D., Russeil D. et al. 2010, A\&A, 518, L101

Zinnecker H., Yorke H. W. 2007, ARA\&A, 45, 481 